Ophthalmologe $2022 \cdot 119: 643-646$ https://doi.org/10.1007/s00347-021-01512-3

Eingegangen: 2. September 2021 Überarbeitet:21. September 2021 Angenommen: 24. September 2021 Online publiziert: 19. Oktober 2021

๑ Der/die Autor(en) 2021

\section{Einseitig rezidivierender Anstieg des Intraokulardrucks mit Hypopyon}

\author{
L. Menghesha' (D) · B. Kirchhof' · R. S. Grajewski' • U. Drebber² · C. Cursiefen ${ }^{1}$. \\ L. M. Heindl ${ }^{1,3}$ \\ 'Zentrum für Augenheilkunde, Medizinische Fakultät und Uniklinik Köln, Universität zu Köln, Köln, \\ Deutschland \\ ${ }^{2}$ Zentrum für Pathologie, Medizinische Fakultät und Uniklinik Köln, Universität zu Köln, Köln, Deutschland \\ ${ }^{3}$ Centrum für Integrierte Onkologie (CIO) Aachen-Bonn-Köln-Düsseldorf, Köln, Deutschland
}

\section{Anamnese und Vorgeschichte}

Eine 86 Jahre alte Patientin wurde mit rezidivierendem Anstieg des Intraokulardrucks (IOD) und Uveitis unklarer Genese des linken Auges bei zunehmender Befundverschlechterung unserer UveitisSprechstunde zugewiesen. Die Patientin berichtete über eine fortschreitende, schmerzlose Visusminderung links, welche im Januar begonnen hätte. Bei Verdacht auf eine glaukomatozyklitische Krise im Sinne eines Posner-SchlossmanSyndroms erfolgte eine externe Vorstellung im April des gleichen Jahres, wobei zunächst eine Vorderkammerpunktion mit anschließender Katarakt-Operation und bei ausbleibendem Therapieansprechen und persistierender Erhöhung des IOD eine Trabekulektomie durchgeführt wurden. Die genannten Eingriffe erfolgten innerhalb von 14 Tagen. Zu diesem Zeitpunkt lagen weder eine dokumentierte Raumforderung der Iris oder des Ziliarkörpers noch

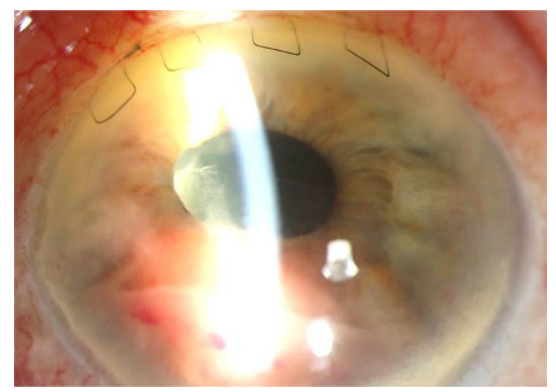

Abb. $1 \Delta$ Vorderabschnittsfoto des linken Auges: Irishyperämie mit sektorieller Raumforderung bei 6 Uhr, Pseudohypopyon und Fibrinreaktion auf der Intraokularlinse die im Verlauf diagnostizierte exsudative Totalamotio vor. In der mikrobiologischen und virologischen Diagnostik wurde kein Erreger nachgewiesen. Die Patientin wurde 20 Tage nach der zuletzt erfolgten Intervention in unserer Poliklinik vorstellig. Im Rahmen des externen Aufenthalts wurde keine tumorspezifische Diagnostik durchgeführt.

\section{Befund}

Der bestkorrigierte Fernvisus betrug rechts 0,5 und links Handbewegungen. Der IOD lag rechts bei $20 \mathrm{~mm} \mathrm{Hg}$ und links bei $14 \mathrm{~mm} \mathrm{Hg}$. Die Lokaltherapie am linken Auge erfolgte mit Prednisolonacetat 1\% 6-mal täglich, Moxifloxacin 2-mal täglich und einem Kombinationspräparat

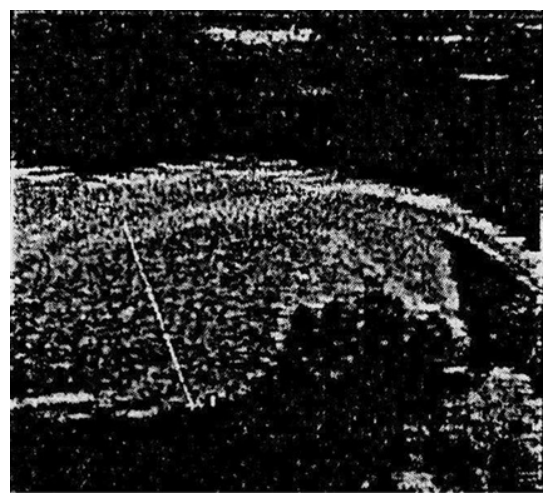

Abb. $2 \Delta$ Ultraschallbiomikroskopie (UBM, ophthalmisches Ultraschallgerät AVISOS, Quantel Medical, Frankreich) des Ziliarkörpers am linken Auge: Raumforderung von 6 bis 8 Uhr mit maximal 5,7 mm Durchmesser. Messung ohne Skleradicke 

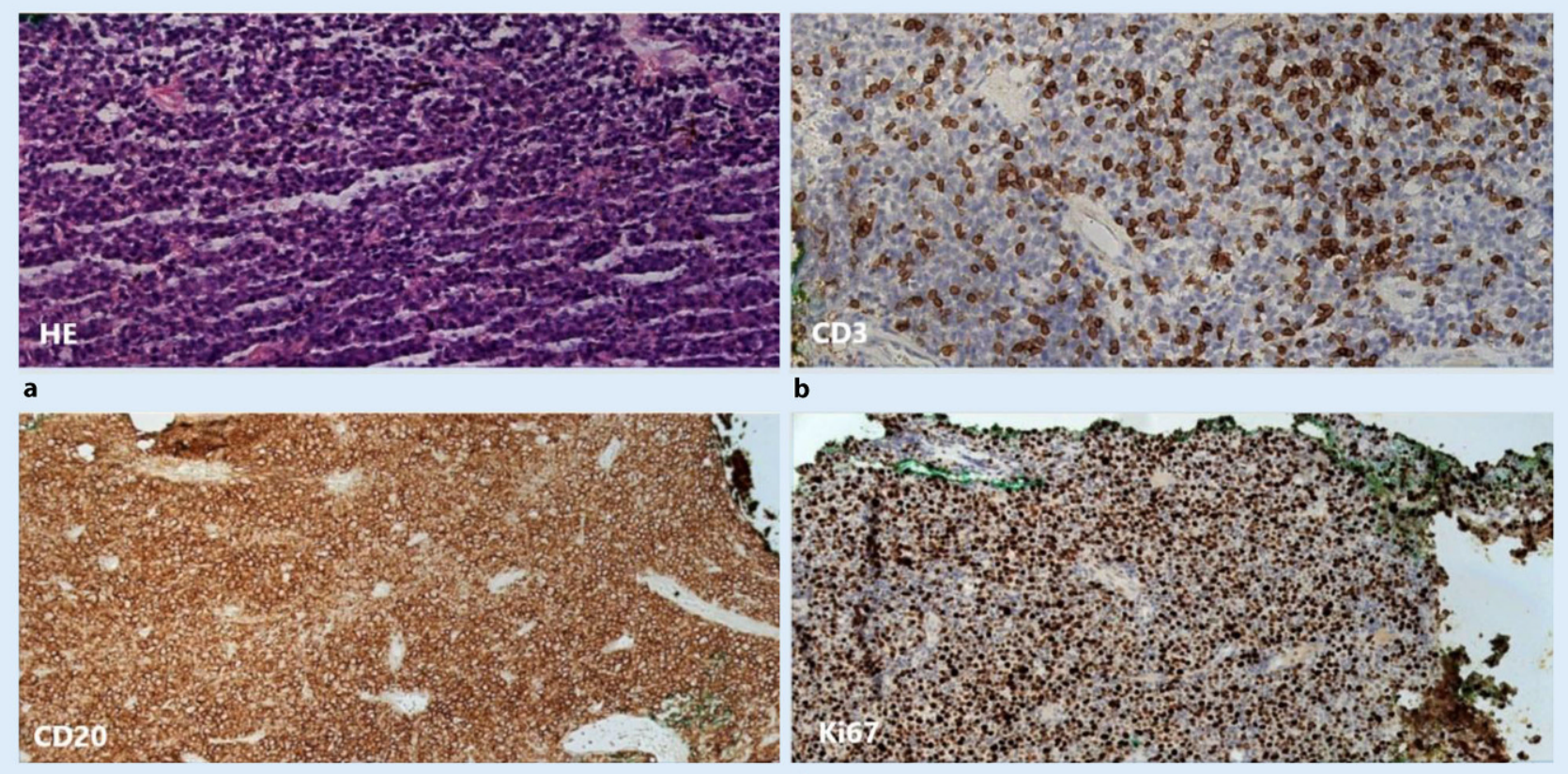

c

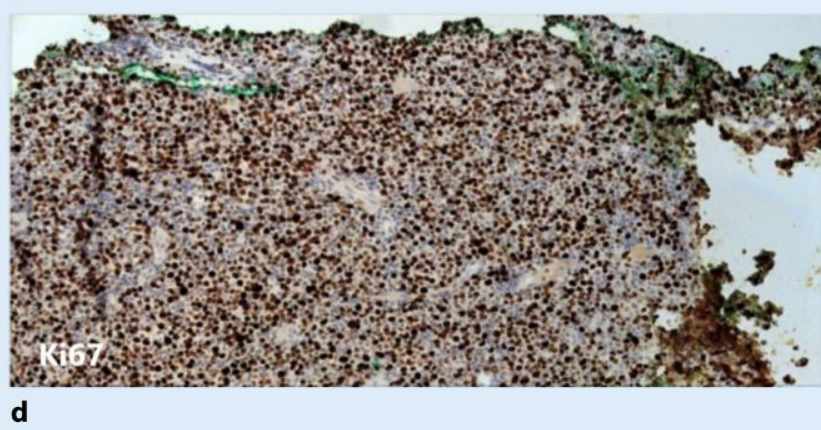

Abb. $3 \Delta$ Histopathologie und Immunhistochemie der Biopsie der Iris des linken Auges bei primärem Irislymphom (diffus großzelliges B-Zell-Lymphom) des linken Auges. a Hämatoxylin-Eosin-Färbung (HE, Vergr. 100:1). Dichte Infiltration durch die Tumorzellen. $\boldsymbol{b}$ Immunhistochemische Färbung gegen CD3 bei begleitender T-lymphozytärer Infiltration (Vergr. 100:1). c Immunhistochemische Färbung gegen CD20 mit kräftiger CD20-Expression der Tumorzellen (Vergr. 100:1). d Immunhistochemische Färbung gegen Ki67 als Proliferationsmarker (Vergr. 100:1)

aus Dorzolamid $20 \mathrm{mg} / \mathrm{ml}$ und Timolol $5 \mathrm{mg} / \mathrm{ml}$ 2-mal täglich. Am rechten Auge lag eine reizfreie Pseudophakie vor. Am linken Auge zeigten sich Hornhautendothelpräzipitate, zweifach positive Zellen, das Tyndall-Phänomen und ein Pseudohypopyon mit einer vaskularisierten Fibrinplatte auf der Intraokularlinse (IOL). Die Iris war hyperämisch und sektoriell von 6 bis 8 Uhr mit deutlicher Prominenz (- Abb. 1).

Am rechten Auge lag die Netzhaut an. Die Papille war vital und randscharf. Am linken Auge lag kein Funduseinblick vor. In der B-Scan-Sonographie präsentierte sich hier eine exsudative Totalamotio. Die Ultraschallbiomikroskopie (UBM) zeigte 5 Wochen nach der Katarakt-Operation eine Raumforderung im Bereich des Ziliarkörpers von 6 bis $8 \mathrm{Uhr}$ mit einem maximalen Durchmesser von $5,7 \mathrm{~mm}$ ohne Skleradicke (• Abb. 2).

Die Patientin wurde zur Vitrektomie mit Retinotomie, IOL-Explantation sowie Vorderkammer-und Glaskörperproben stationär aufgenommen. Intraoperativ erfolgte eine diagnostische Biopsie der Raumforderung mittels Sektoriridektomie bei $6 \mathrm{Uhr}$, sodass keine Andoiridektomie angelegt wurde. Als Endotamponade wurde auf- grund der exsudativen Totalamotio Silikonöl verwendet (Siluron ${ }^{\circledR}$ 2000).

\section{Pathologischer Befund}

Histopathologisch war das exzidierte Gewebe nahezu vollständig eingenommen von einem atypischen nichtkohäsiven, blastenreichen lymphoiden Infiltrat, bestehend aus dicht gelagerten Zellen mit mittelgroßen rundlichen Kernen (• Abb. 3). In dem Infiltrat imponierten mehrere Gefäße.

Wie lautet Ihre Diagnose?

\section{Immunhistochemischer Befund}

Die Immunhistochemie (• Abb. 3) zeigte ein blastoides B-lymphozytäres Infiltrat (CD20-positiv) bei kräftiger Positivität für BCL2 (engl. B-cell lymphoma 2) mit einer hohen proliferativen Aktivität (Ki67 $60 \%$ ), sodass bei alleiniger Tumormanifestation in dieser Lokalisation die Diagnose eines diffus großzelligen B-Zell-Lymphoms des Ziliarkörpers mit Irisbeteiligung gestellt wurde. Eingestreut fanden sich begleitende deutlich kleinere T-Lymphozyten mit Expression von CD3 und CD5 und abschnittsweise HGAL (engl. human germinal center-associated lymphoma).

\section{Weiterer Verlauf}

Zum Staging und interdisziplinären Therapieeinleitung folgte die Vorstellung in der Hämatoonkologie. Hierbei wurden eine umfassende laborchemische Untersuchung des Bluts, eine körperliche Untersuchung und Sonographie des Abdomens durchgeführt. Die kontrastmittelgestützte 
Magnetresonanztomographie (MRT) war ohne Anhalt für einen intrakraniellen oder anderweitig lokalisierten Malignombefall. Im ophthalmoonkologischen Tumorboard wurde nach Ann Arbor Stage System ein Stadium 1EA definiert. Es handelte sich um einen solitären extranodalen Befall ohne B-Symptomatik.

\section{\) Diagnose: Primäres Ziliarkörperlymphom mit Irisbeteiligung (diffus großzelliges B-Zell-Lymphom)}

Es erfolgte auf Wunsch der Patientin eine Monotherapie des CD-20-Antikörpers Rituximab im 3-wöchigen Rhythmus über 12 Zyklen mit Erhaltungstherapie im 8-wöchigen Rhythmus über 2 Jahre. Hierunter zeigte sich in der MRT ein partielles Ansprechen. Eine B-Symptomatik lag zu keinem Zeitpunkt vor. Die ophthalmologischen Kontrollen erfolgten in 6-monatigem Rhythmus. Der Visus lag weiterhin bei Handbewegungen bei normwertigem IOD. Die Enukleation des Auges und eine perkutane Strahlentherapie waren in Anbetracht des erhöhten Lebensalters und fehlenden Schmerzen nicht gewünscht. Die intravitreale Injektion mit Rituximab war bei ausgeprägter Schrankenstörung und Silikonöltamponade sowie stabilen Befunden unter systemischer Therapie nicht indiziert.

\section{Diskussion}

Intraokuläre Lymphome sind eine heterogene Gruppe seltener, lymphoproliferativer Neoplasien und werden nach ihrer anatomischen Lokalisation klassifiziert. Primär vitreoretinale Lymphome sind von uvealen Lymphomen zu differenzieren. Letztere sind selten, treten meistens unilateral auf und können die Aderhaut, die Iris und den Ziliarkörper befallen. Nach chirurgischen Manipulationen kann es zur extraokulären Ausdehnung des Lymphoms kommen [2-5]. Der in diesem Fall beschriebene IODAnstieg kann durch die Störung der BlutKammerwasser-Schranke mit folglich erhöhtem Plasmaproteingehalt in der Vorderkammer bedingt sein. Dies könnte akut und chronisch zu einer Obstruktion des Abflusses über das Trabekelmaschenwerk geführt haben. Außerdem lag neben ei- ner intensiven, steroidhaltigen Lokaltherapie wegen des Uveitisverdachts aufgrund der Raumforderung auch eine mechanische Obstruktion vor.

Aufgrund des seltenen Auftretens der beschriebenen Tumorentität gibt es zwar aktuelle Expertenempfehlungen von Kakkassery et al., jedoch bisher noch kein standardisiertes Vorgehen. In der genannten Publikation werden die Lymphome abhängig von ihrer Morphologie klassifiziert, sodass der hier beschriebene Tumor bei dominanter Ausprägung im Bereich des Ziliarkörpers als primäres Ziliarkörperlymphom mit Irisbeteiligung definiert wird. Der Goldstandard zur Diagnosesicherung ist die Gewebebiopsie mit histopathologischer und immunhistochemischer Aufarbeitung. Anschließend folgt ein Staging durch die Hämatoonkologie mit Durchführung einer laborchemischen Untersuchung des Bluts, körperlichen Untersuchung, Sonographie des Abdomens, MRT oder Computertomographie (CT) von Kopf, Hals, Thorax und Abdomen sowie einer Knochenmarkpunktion und gegebenenfalls Positronenemissionstomographie (PET). Im interdisziplinären Tumorboard wird ein individuelles Therapiekonzept erarbeitet. Häufig beinhaltet es eine Kombination aus systemischer Chemotherapie, Checkpointinhibitoren und Immuntherapie sowie bei visusbedrohenden Befunden einer Strahlentherapie mit 40-80 Gy. Chirurgische Optionen sind intraokuläre Injektionen mit Methotrexat oder Rituximab sowie die Enukleation des Auges. Die erste ophthalmologische Kontrolle sollte nach 6 Wochen erfolgen. Anschließend beträgt das empfohlene Kontrollintervall im ersten Jahr 3 Monate und im zweiten Jahr 6 Monate [1, 4].

\section{Fazit für die Praxis}

- Primäre Ziliarkörperlymphome mit Irisbeteiligung sind selten.

- Die Biopsie des Befundes gilt als Goldstandard zur Diagnosesicherung.

- Ein interdisziplinäres Tumorboard ist zur Therapieplanung unerlässlich.

- Definitive Therapieoptionen umfassen die Strahlentherapie, Immuntherapie mit Rituximab, Chemotherapie und Enukleation des betroffenen Auges.
Korrespondenzadresse

\section{Menghesha}

Zentrum für Augenheilkunde, Medizinische Fakultät und Uniklinik Köln, Universität zu Köln Köln, Deutschland

leonie.menghesha@uk-koeln.de

Funding. Open Access funding enabled and organized by Projekt DEAL.

\section{Einhaltung ethischer Richtlinien}

Interessenkonflikt. L. Menghesha, B. Kirchhof, R.S. Grajewski, U. Drebber, C. Cursiefen und L.M. Heindl geben an, dass kein Interessenkonflikt besteht.

Für diesen Beitrag wurden von den Autoren keine Studien an Menschen oder Tieren durchgeführt. Für die aufgeführten Studien gelten die jeweils dort angegebenen ethischen Richtlinien. Für Bildmaterial oder anderweitige Angaben innerhalb des Manuskripts, über die Patienten zu identifizieren sind, liegt von ihnen und/oder ihren gesetzlichen Vertretern eine schriftliche Einwilligung vor.

Open Access. Dieser Artikel wird unter der Creative Commons Namensnennung 4.0 International Lizenz veröffentlicht, welche die Nutzung, Vervielfältigung, Bearbeitung, Verbreitung und Wiedergabe in jeglichem Medium und Format erlaubt, sofern Sie den/die ursprünglichen Autor(en) und die Quelle ordnungsgemäß nennen, einen Link zur Creative Commons Lizenz beifügen und angeben, ob Änderungen vorgenommen wurden.

Die in diesem Artikel enthaltenen Bilder und sonstiges Drittmaterial unterliegen ebenfalls der genannten Creative Commons Lizenz, sofern sich aus der Abbildungslegende nichts anderes ergibt. Sofern das betreffende Material nicht unter der genannten Creative Commons Lizenz steht und die betreffende Handlung nicht nach gesetzlichen Vorschriften erlaubt ist, ist für die oben aufgeführten Weiterverwendungen des $\mathrm{Ma}$ terials die Einwilligung des jeweiligen Rechteinhabers einzuholen.

Weitere Details zur Lizenz entnehmen Sie bitte der Lizenzinformation auf http://creativecommons.org/ licenses/by/4.0/deed.de.

\section{Literatur}

1. Aronow ME, PortellCA, Sweetenham JWetal(2014) Uveal lymphoma: clinical features, diagnostic studies, treatment selection, and outcomes. Ophthalmology 121:334-341

2. Cursiefen C, Holbach LM, Lafaut B et al (2000) Oculocerebral non-Hodgkin's lymphoma with uveal involvement: development of an epibulbar tumor after vitrectomy. Arch Ophthalmol 118:1437-1440

3. Doycheva D, Zierhut M, Süsskind D et al (2015) Diagnostik und Therapie der choroidalen Lymphome. Ophthalmologe 112:217-222

4. Kakkassery V, Coupland SE, HeindI LM (2020) Iris lymphoma - a systematic guide for diagnosis and 


\section{In eigener Sache}

treatment. Surv Ophthalmol. https://doi.org/10 1016/j.survophthal.2020.06.003

5. Mashayekhi A, Shukla SY, Shields JA et al (2014) Choroidal lymphoma: clinical features and association with systemic lymphoma. Ophthalmology $121: 342-351$

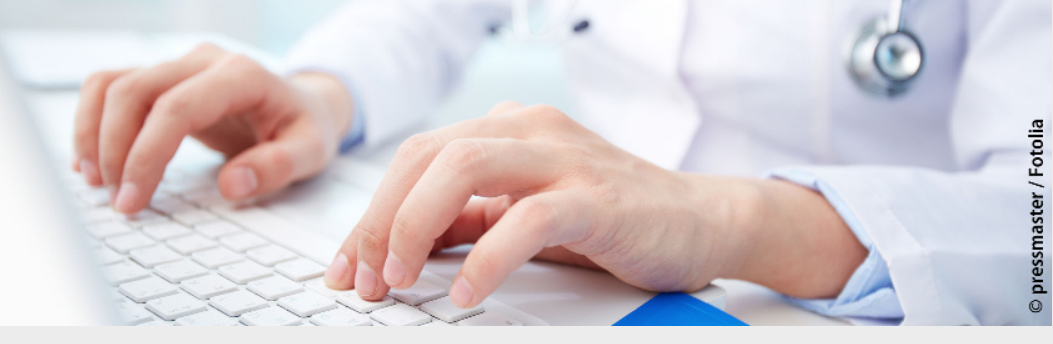

Für Autorinnen und Autoren: Ihr Beitrag in Die Ophthalmologie Das sollten Sie bei Ihrer Einreichung beachten

Wir freuen uns über jede Einreichung in den Rubriken „Originalien“, „Übersichten“, "Kasuistiken" und „Bild und Fall".

Bitte beachten Sie bei der Erstellung Ihres Beitrags folgende Formatvorgaben:

\section{Originalien}

- Vorstellung und Diskussion von Methoden und Ergebnissen eigener Studien

- Gliederung: Hintergrund, Methoden, Ergebnisse, Diskussion

- max. 25.000 Zeichen inkl. Leerzeichen (Literatur, Tabellen und Abbildungslegenden bitte mitzählen)

- Kurze gegliederte Zusammenfassung (deutsch und englisch)

- 5 Schlüsselwörter (deutsch und englisch)

- Max. 6 Abbildungen inkl. Verweise im Text

- Max. 30 Literaturstellen

\section{Übersichten}

- Kritische Zusammenstellung und Diskussion relevanter wissenschaftlicher Ergebnisse

- max. 25.000 Zeichen inkl. Leerzeichen (Literatur, Tabellen und Abbildungslegenden bitte mitzählen)

- Kurze Zusammenfassung (deutsch und englisch)

- 5 Schlüsselwörter (deutsch und englisch)

- Max. 6 Abbildungen inkl. Verweise im Text

- Max. 30 Literaturstellen

\section{Kasuistiken}

- Kurzes Fallbeispiel, das Besonderheiten der klinischen Praxis, Fallstricke der Diagnostik und ungewöhnliche Krankheits- und Behandlungsverläufe aufzeigt

- Gliederung: Anamnese, Befund, Diagnose, Therapie und Verlauf, Diskussion

- max. 10.000 Zeichen inkl. Leerzeichen (Literatur, Tabellen und Abbildungslegenden bitte mitzählen)

- ohne Zusammenfassung und Schlüsselwörter

- Max. 6 Abbildungen inkl. Verweise im Text

- Max. 10 Literaturstellen

\section{Bild und Fall}

- Kurzes Fallquiz, bei dem der Leser miträtseln kann

- Gliederung: Teil 1 - Falldarstellung mit Anamnese, klinischem Befund und Diagnostik. Teil 2 - Diagnose, Therapie und Verlauf, ggf. weitere Hintergrundinformationen

- max. 10.000 Zeichen inkl. Leerzeichen (Literatur, Tabellen und Abbildungslegenden bitte mitzählen)

- ohne Zusammenfassung und Schlüsselwörter

- Max. 5 Abbildungen inkl. Verweise im Text

- Max. 5 Literaturstellen

Ausführliche Leitfäden finden Sie unter https://www.springer.com/journal/347 